This is the author's version of an article that has been published in the ICIT 2019 proceedings.

Changes were made to this version by the publisher prior to publication.

The final version of record is available at https://dx.doi.org/10.1109/ICIT.2019.8755240

\title{
Cost-Optimized Control of DC Microgrids based on Characteristic Diagrams
}

\author{
Elias Knöchelmann, Svenja Tappe, Tobias Ortmaier \\ Gottfried Wilhelm Leibniz Universität Hannover \\ Institute of Mechatronic Systems \\ Hannover, Germany \\ Email: Elias.Knoechelmann@imes.uni-hannover.de
}

Alexander Männel

Bosch Rexroth AG

Lohr am Main, Germany

\begin{abstract}
This paper presents a novel approach for an energy control of a DC microgrid. It combines decentralized grid management and energy management. For this purpose, the conventional voltage droop curves are extended to a characteristic diagram with electricity costs as a further dimension. The support points of these characteristic diagrams are then optimized with a particle swarm optimizer. The target criterion of this optimization is a monetary cost function, that takes several effects, such as depth of discharge, on the operating costs into account. The optimized characteristic diagrams are designed more robust by a sensitivity analysis. The proposed method has been tested successfully in simulations and experiment and was always more cost-efficient than the initial characteristics diagram.

Index Terms-energy control, DC microgrid, characteristic diagrams, voltage droop control
\end{abstract}

\section{INTRODUCTION}

The turnaround in energy policy is one of the major current challenges for the manufacturing industry in Germany. In addition to the creation of an efficient energy infrastructure at a supraregional level, local efforts are required by every energy user. Increasing energy efficiency in production, a higher flexibility to respond to fluctuating energy supplies, and increasing the robustness of production to varying energy supply quality are tasks that must be intensified in the near future.

In the industrial environment the $400 \mathrm{~V}$ three-phase power supply is the standard. The rotational speed of rotary field machines, for this type of power supply system, depends on the mains frequency of the power supply. So converter systems are used to vary the frequency of the motor field and change the motors speed. The converter systems consist of a rectifier which supplies a DC voltage intermediate circuit, and a threephase pulse inverter, providing the rotating field for the motor. Since the rectifiers are usually designed as diode bridges and not as active front ends, no feedback to the $400 \mathrm{~V}$ three-phase system is possible.

By replacing the three-phase grid with a DC grid, in industrial environments, it is possible to exchange electrical energy within the entire factory, which allows the use of a central energy storage at the factory level. Electrical energy generated by braking processes no longer has to be converted into heat in brake resistors, but can be distributed to other systems. The decentralized rectifiers are eliminated, resulting in space

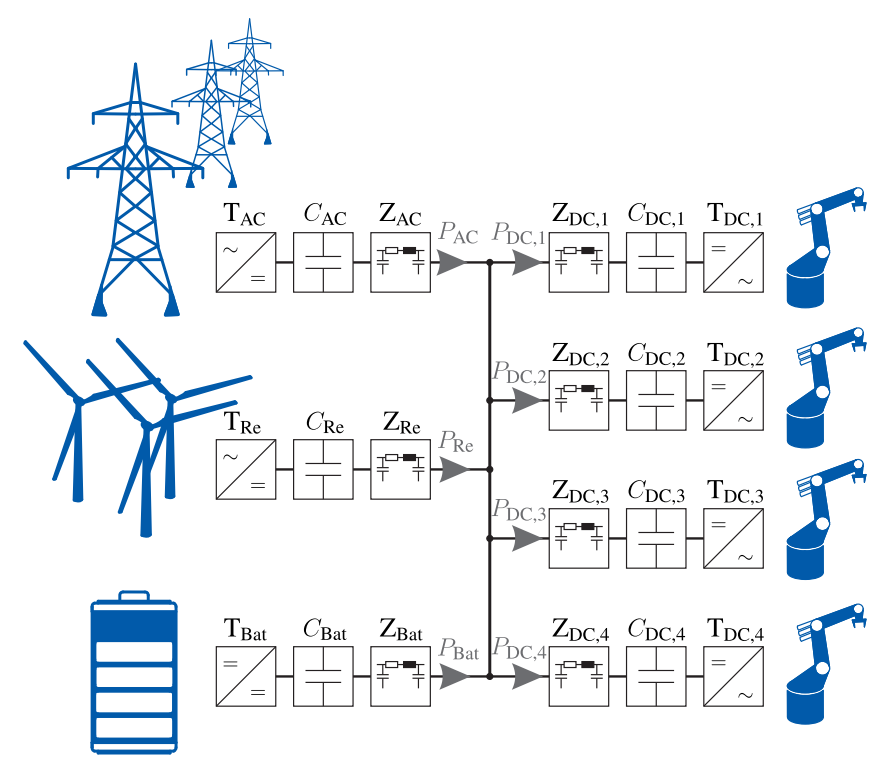

Fig. 1. Overview images of the investigated DC microgrid

savings. The ability to integrate electrically commutated DC motors (EC motors) is increased. These are characterized by their high energy density and their ease of manufacture.

A typical DC microgrid is shown in Fig. 1. The supply is shown on the left side of the grid and consumer are on the right side. The supplier consists of an active front end converter which supplies the grid from the main utility grid $\mathrm{T}_{\mathrm{AC}}$. In addition to the rectifier, the intermediate circuit capacity $C_{\mathrm{AC}}$ of this station can be seen in Fig. 1. The grid participants are connected by a cable. These are shown by the cable equivalent circuit $\mathrm{Z}_{\mathrm{AC}}$ diagram. Fig. 1 shows, that renewable energy source, such as wind turbines or photovoltaic systems, can as well be considered in microgrid systems. An energy storage system can save temporary energy overproduction and, in case of a main supply error, can be used as an uninterruptible power supply. These three suppliers are controlling the grid voltage through their power input. The consumer power (shown on the right side of Fig. 1), on the other hand, is fixed and can't be adjusted as the production process itself is unmodifiable. Therefore all network users whose power consumption cannot 
This is the author's version of an article that has been published in the ICIT 2019 proceedings.

Changes were made to this version by the publisher prior to publication.

The final version of record is available at https://dx.doi.org/10.1109/ICIT.2019.8755240

be changed are hereinafter referred to as passive grid users and whose power consumption can be changed are referred to as active grid users.

The control concepts of DC microgrids can be divided into two categories: central approaches with communication between the participants and decentral approaches without communication. Central approaches are not easy to expand, have high computing time and poor reliability in case of failure of a single DC user [1]. Therefore, this paper presents a decentralized approach. Decentralized DC microgrid control also has some weaknesses, such as suboptimal fulfillment of the control strategy [2], but is a valid alternative to the central approach [1].

Both control concepts have two different focuses. The first, grid management, has the focus to keep the grid voltage within a tolerance range and ensures that all network users are in a suitable operating state. The second method, called energy management, focuses on energy consumption reduction or in the considered case monetary cost reduction of the DC microgrid.

\section{A. Grid Management}

The regulation of the DC mains voltage must be controlled very quickly. Therefore decentralized approaches are often used [3]. Popular options are adaptive droop voltage [4] or fuzzy control [5]. By using e.g. adaptive droop voltage control, the DC mains voltage can be kept within limits. Some example droop curves are displayed in Fig. 2. In this work, this approach is picked up and expanded.

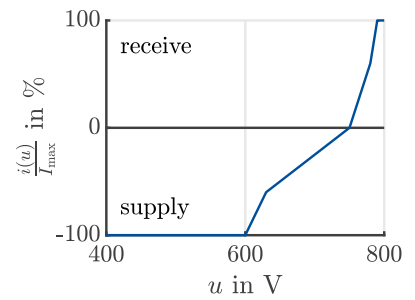

(a) Droop curves of the rectifier

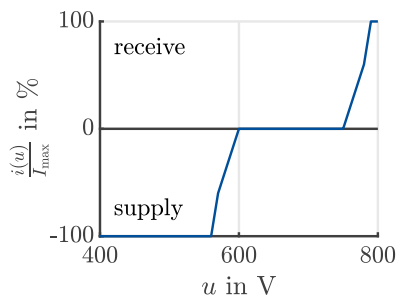

(b) Droop curves of the battery
Fig. 2. Exemplary droop curves for active grid users

The control loop for the trial cell is displayed in Fig. 3. The controller provides the output current depended on the measured DC voltage. Calculating the control deviation by subtracting desired form measured voltage is done in the controller through the droop voltage curves. The output current of the active grid users feeds the DC grid. The passive grid users on the other hand interfere the grid and are, therefore, the disturbance of the control loop.

\section{B. Energy/Cost Management}

Energy management is often implemented via central approaches, since external information such as battery status or electricity price is required [6]. There are different approaches for central control e.g. model predictive [7] or scheduling and a short-time predictive control [8]. Although a central

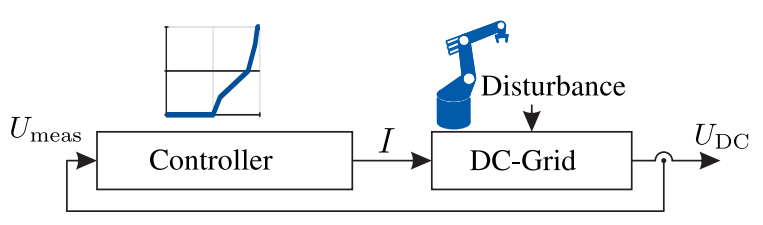

Fig. 3. Block diagram of the trial cell

approach for energy management is appropriate, due to the aforementioned disadvantages, some work has focused on decentralized approaches [9], [10]. A possibility of decentralized energy management with characteristic lines is [9]. Here the curves is adapted online by a genetic algorithm. An essential difference in the literature is the optimization criterion, the cost function. Here often only the electricity costs are used to evaluate the absorbed energy [8]. In manufacturing industry electricity costs are usually fixed, but these get updated every 15 minutes at the electricity stock exchange [11]. There are already electricity rates who offer this fluctuating price [12]. A detailed approach for a cost function can be found in [9] where various operating costs are integrated. However, the discharge depth is neglected, which has a strong influence on the lifetime and thus on the operating costs.

We propose a new approach to achieve this cost efficient grid management concept. First, the voltage droop curves have to be extended to a characteristic diagram with the electricity costs as a further dimension. A particle swarm optimizer is used to simplify and optimize the design of the new diagrams. The entire optimization process is performed offline. As an optimization criterion, a cost function was set up which, among others, takes into account the energy storage service life reduction through usage. To make these characteristic diagram more robust, a sensitivity analysis is applied and the characteristic diagram is revised with respect to the result. In the end, the results are compared and analyzed.

The article is organized as follows: In Section II, the layout of the system is described and the modeling is presented. Moreover the modeling is validated with an experimental setup. The structure of the characteristic diagrams is described in Section III, followed by the formulation of the cost function for the optimization. Based on a sensitivity analysis the final characteristic diagrams are created. The results will be discussed in Section IV. The paper closes with a conclusion in Section V.

\section{SYSTEM DESCRIPTION AND MODELING}

At first we take a look at the trial structure. To really take benefits from the change to a DC microgrid the consumers need to offer a lot of breaking energy to the DC microgrid. Robots have synchronised movements, so all axes of one robot are breaking at the same time and a lot of breaking energy is generated, which is normally converted into heat. By establishing a DC microgrid this energy can be distributed to other robots. Due to the outlined benefits, the DC microgrid considered in this paper is an exemplary robot cell. Its design and modeling will be presented in this section. 
This is the author's version of an article that has been published in the ICIT 2019 proceedings.

Changes were made to this version by the publisher prior to publication.

The final version of record is available at https://dx.doi.org/10.1109/ICIT.2019.8755240

\section{A. Trial Cell}

The trial cell is composed by four KUKA KR 16-2 robots as seen in Fig. 1. As shown in previous work [13], the energy consumption of each robot can be simulated with high accuracy. As renewable energy source a photo voltaic system was added. For interruptibility power supply purposes a generic lithium-ion battery was included into the grid. The supply is realized as an active front end converter.

For the optimization, a randomized data set was generated for each robot covering two times 50 point to point movements. The power curves of these four robots were merged together with different pause lengths. The data for the photo voltaic system are measurements originated from a private facility. To increase the variance in the data, measurements were scaled down from 2.5 days to 2 hours. This also has the effect that a more complex test scenario has been created. The power curve used for optimization is shown in Fig. 4. The electricity price change was reduced from 15 minutes to 5 minutes for the same reason as mentioned previously. Its change was modeled based on a real price of electricity course on the stock exchange in Germany [11]. In order to show that the optimization results are not only valid for the power curve used for optimization, a validation power curve with the same criteria was generated.

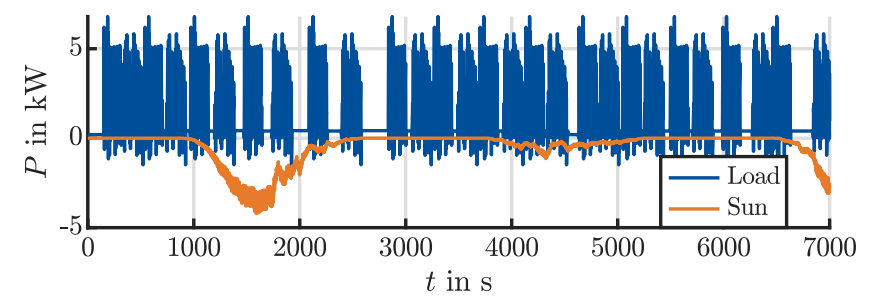

Fig. 4. Representation of the power curves which were used for optimization.

\section{B. DC-Grid Modeling}

For optimization proposes a fast model needs to be designed. This is done by neglecting cable losses. Each producer and consumer has a huge DC link capacity. Compared to these capacities the cables losses, for short cables, have nearly no effect. Without cables losses the DC link capacities can be merged into one capacity. The general idea behind the DC grid modeling is that the energy $E$ stored in the electric field of a capacitor can be described by:

$$
E=\frac{1}{2} \cdot C \cdot U^{2}
$$

with $C$ being the capacity of a general capacitor and $U$ being the applied electrical voltage, utilized to our case, $U$ is the grid voltage. The controller output $I$, which dependents on the DC gird voltage, needs to be converted to DC grid power by multiplying it with the DC grid voltage. The DC link power of active users can be calculated on this way, the DC grid power of passive users are given as a load profile or can be measured. By multiplying the DC grid power $P_{\text {sum }}$ of each
DC grid users by the time step $\Delta T$ used in the simulation the realized work $\Delta W_{\text {sum }}$ in time step $k$ is received

$$
\Delta W_{\text {sum }}(k)=P_{\text {sum }}(k) \cdot \Delta T .
$$

Under the assumption, that the realized work $\Delta W_{\text {sum }}$ is stored in the electrical field, Eqn. 1 can be rewritten as:

$$
U_{\mathrm{DC}}(k)=\sqrt{\frac{2}{C_{\mathrm{DC}}} \cdot\left|\Delta W_{\text {sum }}(k)\right|+U_{\mathrm{DC}}^{2}(k-1)} .
$$

Whereas $C_{\mathrm{DC}}$ is the DC link capacity and $U_{\mathrm{DC}}(k)$ is the resulting DC grid voltage for the time step $k$. With this equation the DC voltage can be calculated based on the consumer power.

\section{Model Evaluation}

The model is compared with a test setup consisting of two active front ends and a DC-DC converter with a lithium-ion battery. Fig. 5 shows a comparison of the qualitative model and the measured values from the test setup. The measured DC grid voltage is obtained through the internal voltage sensor of the active front end, the DC grid power by multiplication of voltage and current sensor data. The grid voltage is well approximated by the model, considering how noisy $( \pm 5 \mathrm{~V}$ see Fig. 5) the voltage measurement is. The power measurement also fits the model prediction. The power peaks are slightly overestimated and the constant interval following the peaks are somewhat underestimated, but the energy error between measured and simulated energy is $4.5 \%$ with a measurement duration of 16 minutes. Fig. 5 shows only the first minute of the evaluation, since the other 15 minutes show no differences in behavior.

The test setup is not part of this paper and will be discussed in future work [14].

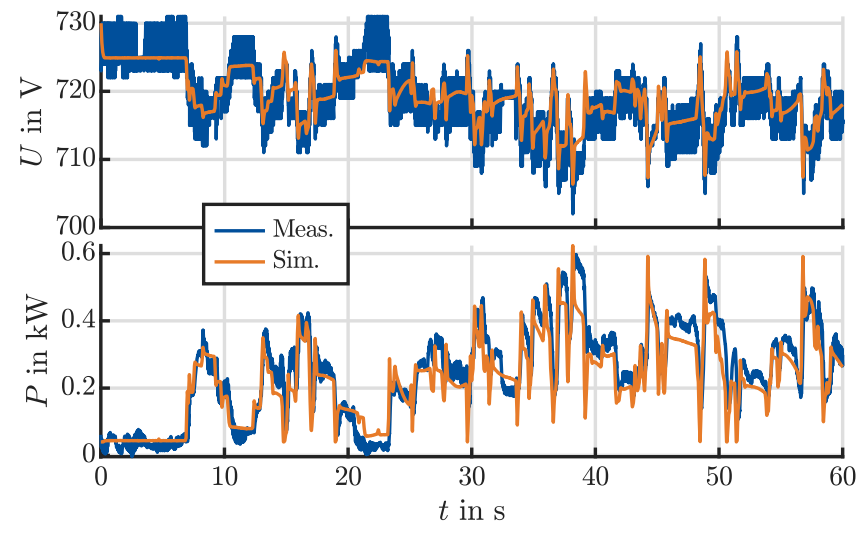

Fig. 5. Comparison of the proposed model with a measurement of the test setup.

\section{OPTIMIZATION}

In this section the optimization of the controller's behavior is addressed. For this purpose the adaptive droop voltage control approach, previously proposed by [15], is extended by an additional dimension. The grid points of the characteristic diagram are then optimized using a monetary cost function. 
This is the author's version of an article that has been published in the ICIT 2019 proceedings.

Changes were made to this version by the publisher prior to publication.

The final version of record is available at https://dx.doi.org/10.1109/ICIT.2019.8755240

\section{A. Characteristic Diagram}

The behavior of the DC grid needs to be adapted depending on the electricity price, therefore, it is added as further dimension to the characteristic diagram. The characteristic diagram is defined by 45 support points: nine voltage steps ( $400 \mathrm{~V}$ to $800 \mathrm{~V}$ in $50 \mathrm{~V}$ steps) and 5 cost steps (-10, 0, 10, 100 and 1000 $\frac{\text { Euro }}{\text { MWh }}$ ) These voltage steps and cost steps are fixed. At each of these support points an adequate feeding current $I$ must be determined. This set-up has convergence reasons, because dependencies of parameter are reduced. Before starting, the optimization initial diagrams for the active front end (Fig. 6(a)) and for the storage (Fig. 6(b)) are defined. The voltage $u$ in $\mathrm{V}$ is plotted on the $x$-axis, the electricity costs $K$ in $\frac{\text { Euro }}{\text { MWh }}$ on the $y$-axis and the current $i(u, K)$ in \% normalized to the maximum value $I_{\max }$. The initial diagrams (Fig. 6) were designed as an approximation of the diagrams in Fig. 2. The working voltage of the DC grid was set to $650 \mathrm{~V}$ and not 750 $\mathrm{V}$ as in Fig. 2.

It is important to notice, that in Fig. 6(a) the current values for electricity costs above $1000 \frac{\text { Euro }}{\mathrm{MWh}}$ are set to 0 , because it can be assumed that such values are equivalent to a power failure. This feature can later be used to ensure uninterruptible power supply.
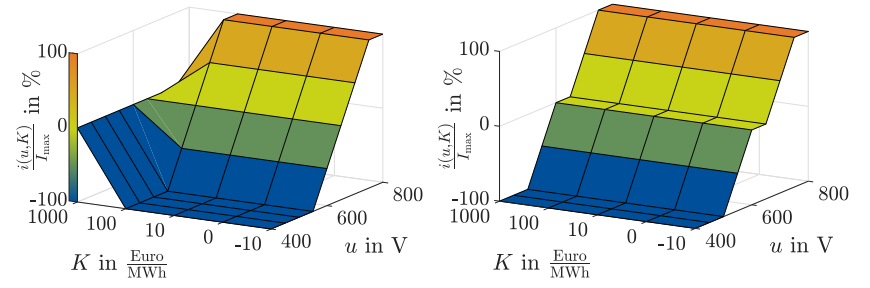

(a) Initial characteristic diagram for (b) Initial characteristic diagram for AFE control. energy storage control system.

Fig. 6. Display of the initial characteristic diagrams.

\section{B. Cost Function}

In order to obtain conclusive optimization results, a cost function standardized to monetary costs was created. This has the advantage that reduction in service life of the energy storage device and also the energy consumption from the threephase network can be taken into account easily. First, the energy consumption $E_{\mathrm{AFE}}$ from the three-phase network can be converted into costs via the electricity price $K$ :

$$
\zeta_{\mathrm{AFE}}=K \cdot E_{\mathrm{AFE}}
$$

The converted energy of the active rectifier $E_{\mathrm{AFE}}$ is calculated by

$$
E_{\mathrm{AFE}}=\int_{t_{0}}^{t_{\mathrm{end}}} P_{\mathrm{AFE}} \mathrm{d} t,
$$

where $P_{\mathrm{AFE}}$ is the converted rectifier power, $t_{0}$ is the point in time at which the electricity costs change and $t_{\text {end }}$ up to which the costs remain constant. Assuming that the lifetime of the energy storage is reduced during discharge/charge, the following costs $\zeta_{\text {bat }}$ for each charging cycle can be calculated with:

$$
\zeta_{\text {bat }}=\frac{K_{\mathrm{inv}} \cdot E_{\mathrm{LI}}}{a \cdot \mathrm{e}^{b \cdot \mathrm{DOD}}}
$$

whereas $K_{\mathrm{inv}}$ is the investment cost for the energy storage, $E_{\mathrm{LI}}$ is the storable energy, $a$ and $b$ are the fitted parameters, that describe the relationship between depth of discharge (DOD) and the cycle reduction. Data on the discharge depth and Lifetime were taken from [16]. The investment cost for lithium-ion batteries was set to $200 \frac{\text { Euro }}{\mathrm{MWh}}$ based on [17]. The energy in the energy storage $E_{\text {bat,stor }}$ at the end of simulation is evaluated with the latest electricity price $K_{\text {end }}$ and included in the cost function. The total costs $\zeta_{\text {tot }}$ are thus calculated as follows:

$$
\zeta_{\text {tot }}=\zeta_{\mathrm{AFE}}+\zeta_{\mathrm{bat}}-K_{\mathrm{end}} \cdot E_{\mathrm{bat}, \mathrm{stor}} .
$$

\section{Optimization Result}

Since the cost function has many local minima a particle swarm optimization (PSO) was used for minimization. The PSO does not guarantee that the global minimum will be found, but an acceptable solution in reasonable time. The results of the optimization can be seen in Fig. 7.

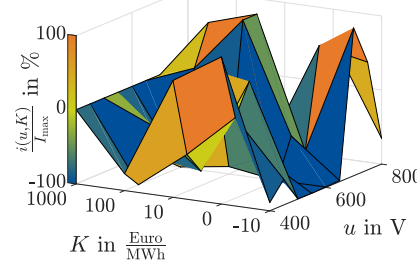

(a) Optimized characteristic diagram for AFE control.

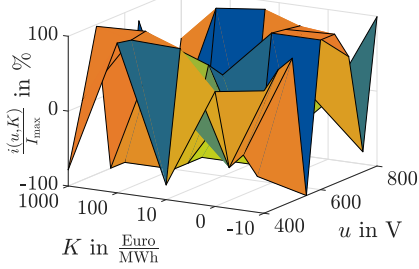

(b) Optimized characteristic diagram for controlling the energy storage system.
Fig. 7. Display of the optimized maps, which were created by the optimization of mesh points.

The diagram looks as if the mesh points have been distributed completely randomly and have not been optimized. However, the costs for the simulation data shown in Fig. 4 have been reduced from over 15 Euro to under zero. Thus, the characteristic diagram is optimally designed for the considered load curve, but if this is no longer the case, it could lead to instabilities due to the high variation. The reason why the characteristic diagram shows such high variation is because these areas have no influence on the cost function. These points have not been excited within the simulation set-up and have, therefore, no influence on the optimization results. In order to make the characteristic diagram more generally valid, the influence of the respective point has on the system will be taken under consideration.

\section{Sensitivity Analysis}

Sensitivity analysis is an economic methodology for assessing how sensitive key figures are to small changes in input parameters [18]. A distinction can be made between 
This is the author's version of an article that has been published in the ICIT 2019 proceedings.

Changes were made to this version by the publisher prior to publication.

The final version of record is available at https://dx.doi.org/10.1109/ICIT.2019.8755240

local and global sensitivity analysis. In the global sensitivity analysis, several input variables are randomly changed and a large number of model runs have to be carried out to obtain significant data. In the local sensitivity analysis, only one input variable is changed by a defined value. If the change of the output $\Delta Y_{i}$ is divided by the input $\Delta X_{i}$, the sensitivity $S_{i}$ in the node $i$ is obtained:

$$
S_{i}=\frac{\Delta Y_{i}}{\Delta X_{i}}
$$

This method is called normalized difference and differential quotient and for reasons of runtime, this method was chosen. The results of the local sensitivity analysis is shown in Fig. 8.

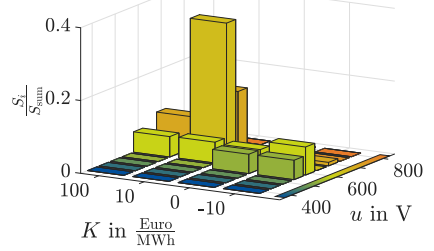

(a) Sensitivity analysis of the characteristic diagram for AFE control.

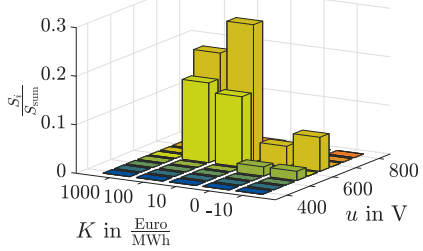

(b) Sensitivity analysis of the characteristic diagram for enery storage control system.
Fig. 8. Sensitivity analysis of the individual nodes to determine the influence of these on the system.

The sensitivity displayed on the $z$-axis has been normalized in total to one. The sensitivity analysis shows which parameters have a large influence on our system costs and must therefore correspond to the optimized parameters. In addition, the analysis shows which parameters have less influence and can therefore be set to any desired value, e.g. all parameters for $500 \mathrm{~V}$ and smaller. But the sensitivity analysis does not include every possible parameter variation. By changing certain parameters, the DC microgrid can reach areas that are not permissible, e.g. resulting DC grid voltage is too high. Since this case of operation is not permitted, it intercepted by constraints and is not valued with cost. Because no result is obtained with this parameter selection, it cannot be included in the sensitivity analysis (Fig. 8). Therefore the triggering of the constraints is used to create parameter limits, which can be seen in Fig. 9.

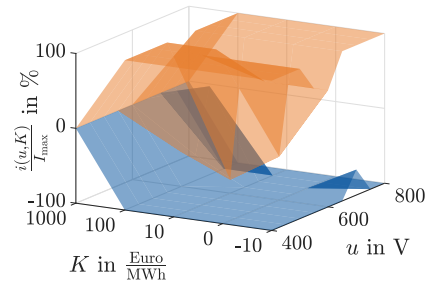

(a) Parameter limits for the characteristic diagram for AFE control.

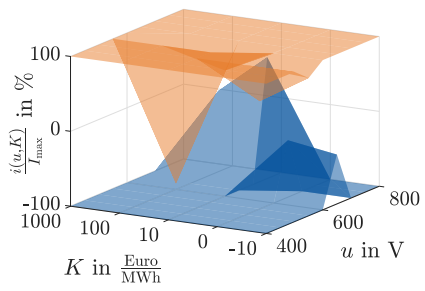

(b) Parameter limits for the characteristic diagram for energy storage system control.

Fig. 9. Tuning limits of the characteristic parameters from the triggering of the constraints in the sensitivity analysis, whereas orange is the upper limit and blue is the lower limit.

The parameter limits show which parameter ranges are permissible and which are not, with orange representing the upper and blue the lower limit.

\section{E. Creation of the Final Characteristic Diagram}

The parameter limits in Fig. 9 can be used to create the final characteristic diagrams. These diagrams can now be designed by hand or with an algorithm automatically. The result of an automatic design can be seen in Fig. 10.

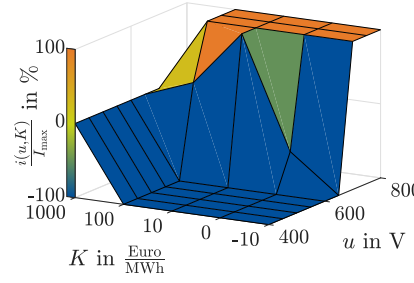

(a) Final characteristic diagram for AFE control.

Fig. 10. Final and robust characteristic diagrams

The basic idea behind the automatic design was that before a value with a high sensitivity (i.e. voltage low), the grid users should only feed energy into the DC grid and after a high sensitivity (i.e. voltage high), energy should be fed back from the DC grid. The automatic design has set the minimum parameter limits before parameter points with high sensitivity and maximum after sensitivity increases. This property can be seen in Fig. 10, because the maximum or minimum parameter limit were often used. In areas of high sensitivity, the optimization results are used, leading to a huge change within the diagram.

\section{RESULTS}

For evaluation reasons, the characteristic shown in Fig. 3 is now replaced by the initial and final characteristic diagram shown in Fig. 10. In a first step, the simulation is performed based on the same data set as used for optimization. In the second step, the characteristic curves are validated on a new data set. In addition, the characteristic diagrams are implemented in the outlined test setup and the result is evaluated.

\section{A. Simulations Results}

In Fig. 11 the upper plot shows the DC grid voltage in blue and the feed-in power of the active rectifier in orange. The plot underneath shows the power curves of the individual network users, using the load curve is shown in Fig. 4. The electricity cost over time is plotted on the right $y$-axis.

The left $y$-axis limit corresponds to the power limit of the AFE $(30 \mathrm{~kW})$ and the energy storage $(30 \mathrm{~kW})$, respectively. Fig. 11 shows that the costs have no influence on the power and voltage curves, because the output current of the initial characteristic diagram is independent of the electric price. The high operation costs (Tab. I) of the initial characteristic diagrams are due to the fact that the energy storage is constantly in use and is constantly charged and discharged. These very 
This is the author's version of an article that has been published in the ICIT 2019 proceedings.

Changes were made to this version by the publisher prior to publication.

The final version of record is available at https://dx.doi.org/10.1109/ICIT.2019.8755240

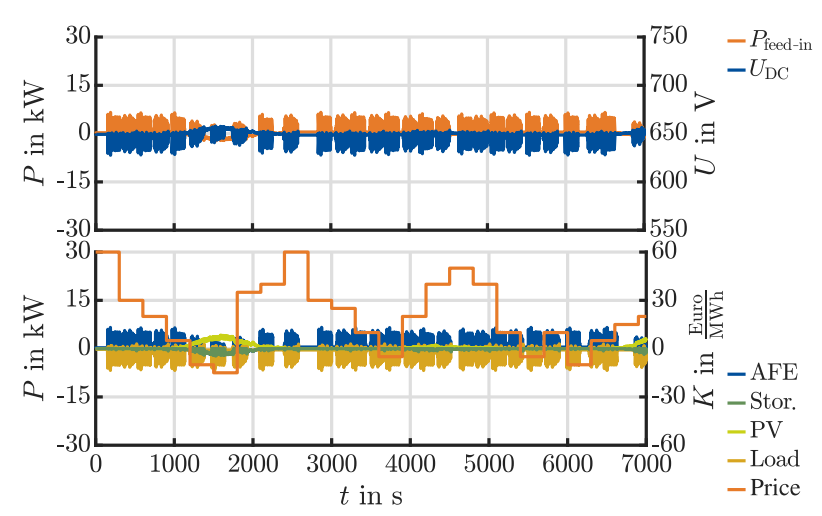

Fig. 11. Plot of the different characteristics of the simulated DC microgrid with the initial characteristic Diagrams

short loading times, often less than $1 \mathrm{~s}$, result in a short service life and thus high operation costs.

Fig. 12 shows the voltage and power curve of the DC grid using the optimized characteristic diagrams. The dependence of the electricity price can be easily recognized: From $1000 \mathrm{~s}$ the electricity price drops below $0 \frac{\text { Euro }}{\mathrm{MWh}}$ and the AFE feeds energy into the DC grid and the storage stores it. As soon as the electricity price becomes high (approx. 2500 s) the AFE feeds the energy back into the three-phase grid. The battery does not change often between charging and discharging, which results in lower running costs compared to Fig 11.

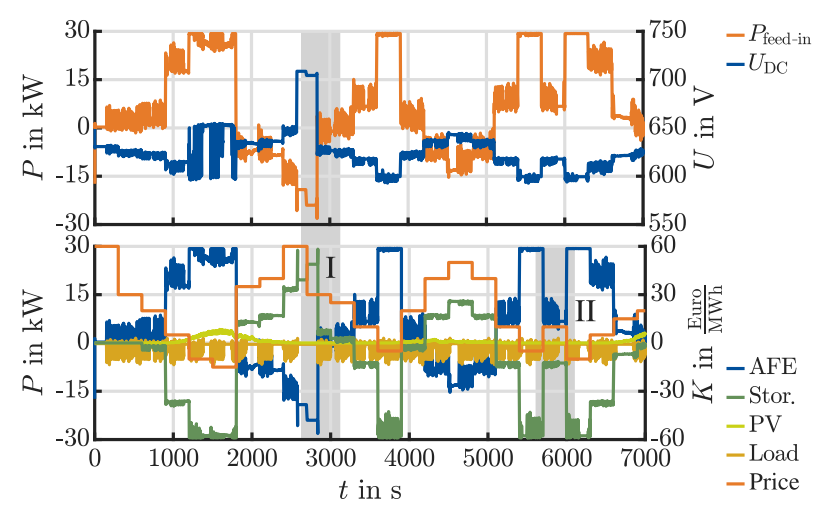

Fig. 12. Plot of the different characteristics of the simulated DC microgrid with the optimized characteristic diagrams

Fig. 12 area I shows that variation in the characteristic diagram can lead to instabilities. Although the electricity costs are lower, the power consumed by the battery increases. However, as soon as the load consumes power, the DC voltage drops and the battery greatly reduces the consumption.

Fig. 13 shows the model outputs with the final characteristic diagrams. Compared to Fig. 12, the feedback from the main grid is lower and therefore the operating costs are minimally higher (Tab. I).

The switching points for charging and discharging the battery have also shifted. It is noticeable that in Fig. 12 area II energy is still pushed into the battery. In Fig. 13 area III,

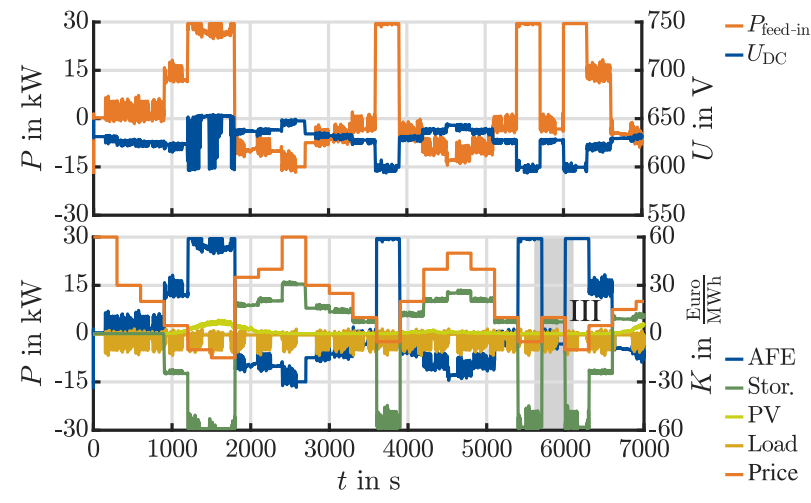

Fig. 13. Plot of the different characteristics of the simulated microgrid with the finalized characteristic diagrams

however, energy is fed back to the main supply. In addition, the event which appear in Fig. 12 area I does not appear in Fig. 13.

Fig. 14 does not use the power profile shown in Fig. 4 but a new validation profile. This can be seen in particular in the fact that photo voltaic power is lower, the load power profiles somewhat higher and the electricity costs only become negative at $5600 \mathrm{~s}$. The plot shown in Fig. 14 was simulated with the final characteristic diagrams. In this process it can be clearly seen that the initial value for the energy storage charge was always zero at the start. Based on this plot, it can be assumed that the final characteristic diagrams not only achieve an improvement on the optimization data set but also with respect to changed conditions.

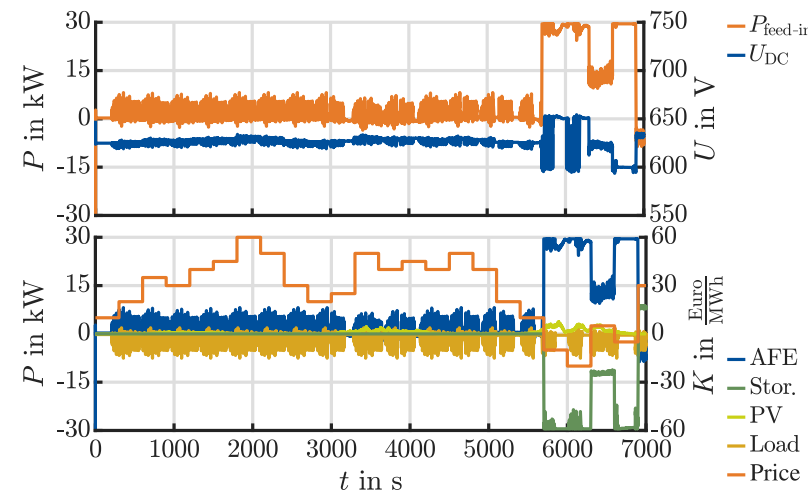

Fig. 14. Plot of the different characteristics of the simulated microgrid with the finalized characteristic diagrams and the validation power curve

A comparison of the simulation results can be found in Tab. I. It can be seen that in both cases the final characteristic diagrams are worse than the characteristic diagrams optimized on the data. In both cases, however, they are better than the initial characteristic diagrams and more robust against changes in the power curves.

\section{B. Experimental Results}

For experimental evaluation, the characteristic diagrams were tested on the test setup, which was used on for the 
This is the author's version of an article that has been published in the ICIT 2019 proceedings.

Changes were made to this version by the publisher prior to publication.

The final version of record is available at https://dx.doi.org/10.1109/ICIT.2019.8755240

TABLE I

COSTS FOR ONE MODEL RUN WITH DIFFERENT CHARACTERISTIC DIAGRAMS (CD)

\begin{tabular}{|c|c|c|c|c|}
\hline applied method & initial CD & opt. CD & final CD & opt. ${ }^{1}$ CD \\
\hline $\begin{array}{c}\text { optimization course } \\
\text { of performance }\end{array}$ & $15.89 €$ & $-0.39 €$ & $-0.29 €$ & - \\
\hline $\begin{array}{c}\text { new course } \\
\text { of performance }\end{array}$ & $20.31 €$ & $-0.10 €$ & $-0.15 €$ & $-0.26 €$ \\
\hline
\end{tabular}

model validation, with the load profiles shown in Fig. 4. The experimental results are showed in Fig. 15.

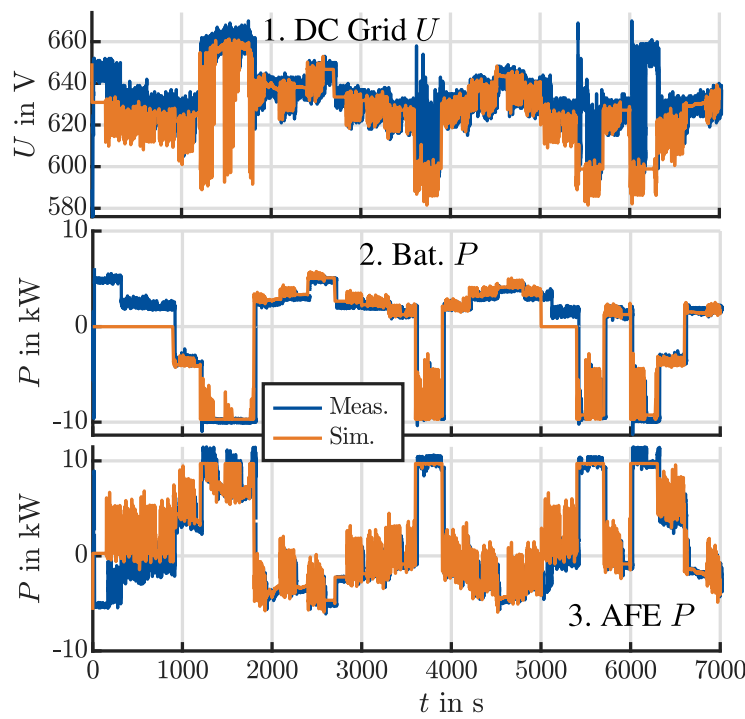

Fig. 15. Plot of the different characteristics of the test setup with the finalized characteristic Diagrams

In the upper plot the DC gird voltage is shown, whereas in the test setup the AFE output voltage was assumed as DC grid voltage. This also explains the large differences between model and measurement at 3800, 5500 and 6100 s. Since the AFE has pushed a lot of power into the DC grid, its voltage is higher than that of the battery which stores energy. In the simplified model there are no different voltages for different grid users.

The performance curves fit together well. Only in the first $1000 \mathrm{~s}$ there is a apparent difference. This is due to the fact that in the simulation the starting value of the battery is $0 \mathrm{Wh}$ and in the experiment is not. The power limit of the AFE and the battery had to be limited to $10 \mathrm{~kW}$ due to line protection.

\section{CONCLUSION}

In this article a very simple modeling for a DC microgrid was presented and validated. The usual droop voltage control in the DC grid range has been extended with respect to the electricity price. The resulting characteristic diagrams were optimized to minimal monetary costs with a particle swarm optimization and post-processed using a sensitivity analysis.

${ }^{1}$ characteristic diagram optimized for the new performance curve
The final characteristic diagrams were then evaluated against each other on the basis of the optimization performance curve and a validation curve and it can be stated that this procedure is more cost effective in both cases than the usual characteristicbased control.

\section{ACKNOWLEDGMENT}

This project was part of the DC-INDUSTRIE research project, funded by the German Federal ministry of Economic Affairs and Energy (BMWi).

\section{REFERENCES}

[1] L. Meng, E. R. Sanseverino, A. Luna, T. Dragicevic, J. C. Vasquez, and J. M. Guerrero, "Microgrid supervisory controllers and energy management systems: A literature review," Renewable and Sustainable Energy Reviews, vol. 60, pp. 1263-1273, 2016.

[2] N. Chatzargyriu, Ed., Microgrids: Architectures and control, onlineausg ed. Chichester, West Sussex, U. K: Wiley, 2014. [Online]. Available: http://onlinelibrary.wiley.com/book/10.1002/9781118720677

[3] B. Wunder, L. Ott, J. Kaiser, Y. Han, F. Fersterra, and M. März, 2015 IEEE First International Conference on DC Microgrids (ICDCM): 7-10 June 2015. Piscataway, NJ: IEEE, 2015. [Online]. Available: http://ieeexplore.ieee.org/servlet/opac?punumber=7139319

[4] L. Ott, Y. Han, B. Wunder, J. Kaiser, F. Fersterra, M. Schulz, and M. Marz, "An advanced voltage droop control concept for grid-tied and autonomous dc microgrids," in 2015 IEEE International Telecommunications Energy Conference (INTELEC). IEEE, 2015, pp. 1-6.

[5] H. Kakigano, Y. Miura, and T. Ise, "Distribution voltage control for dc microgrids using fuzzy control and gain-scheduling technique," IEEE Transactions on Power Electronics, vol. 28, no. 5, pp. 2246-2258, 2013.

[6] D. E. Olivares, A. Mehrizi-Sani, A. H. Etemadi, C. A. Canizares, R. Iravani, M. Kazerani, A. H. Hajimiragha, O. Gomis-Bellmunt, M. Saeedifard, R. Palma-Behnke, G. A. Jimenez-Estevez, and N. D. Hatziargyriou, "Trends in microgrid control," IEEE Transactions on Smart Grid, vol. 5, no. 4, pp. 1905-1919, 2014.

[7] A. Parisio, E. Rikos, and L. Glielmo, "A model predictive control approach to microgrid operation optimization," IEEE Transactions on Control Systems Technology, vol. 22, no. 5, pp. 1813-1827, 2014.

[8] A. Bracale, P. Caramia, G. Carpinelli, E. Mancini, and F. Mottola, "Optimal control strategy of a dc micro grid," International Journal of Electrical Power \& Energy Systems, vol. 67, pp. 25-38, 2015.

[9] C. Li, F. de Bosio, F. Chen, S. K. Chaudhary, J. C. Vasquez, and J. M. Guerrero, "Economic dispatch for operating cost minimization under real-time pricing in droop-controlled dc microgrid," IEEE Journal of Emerging and Selected Topics in Power Electronics, vol. 5, no. 1, pp. 587-595, 2017.

[10] I. U. Nutkani, W. Peng, P. C. Loh, and F. Blaabjerg, "Cost-based droop scheme for dc microgrid: 14 - 18 sept. 2014, pittsburgh, pa, usa," 2014.

[11] European Energy Exchange, "Leipzig electricity stock exchange," 2018. [Online]. Available: https://www.eex.com/de/

[12] Next Kraftwerk, "Variable electricity tariffs for industry \& commerce," 2018. [Online]. Available: https://www.next-kraftwerke.de/virtuelleskraftwerk/stromverbraucher/variabler-stromtarif

[13] K. Eggers, E. Knöchelmann, S. Tappe, and T. Ortmaier, "Modeling and experimental validation of the influence of robot temperature on its energy consumption," vol. 2018, 2018.

[14] A. Männel, S. Tappe, E. Knöchelmann, and T. Ortmaier, "Investigation on an ac grid failure handling of industrial dc micro grids with an energy storage: Submitted to icit 2019," 2019.

[15] D. A. Schaab, S. Weckmann, T. Kuhlmann, and A. Sauer, "Flexible, robust and sustainable energy supply through industrial smart-dc-grid with decentralized grid management," 25th CIRP Life Cycle Engineering (LCE) Conference,, vol. 30 April - 2 May 2018, 2018.

[16] I. Stadler, Energiespeicher: Bedarf, technologien, integration. Berlin: Springer Vieweg, 2014

[17] Horváth \& Partners, "Global price development for lithium-ion batteries from 2013 to 2020," 04.2016. [Online]. Available: https://de.statista.com/statistik/daten/studie/534429/umfrage/weltweitepreise-fuer-lithium-ionen-akkus/

[18] A. Saltelli, Sensitivity analysis in practice: A guide to assessing scientific models, reprinted. ed. Hoboken, NJ: Wiley, 2007. 